Cibele Figueira CARVALHO ${ }^{1}$

Maria Cristina CHAMMAS ${ }^{1}$

Franklin De Almeida

STERMANN ${ }^{2}$

Nestor de BARROS ${ }^{1}$

Giovanni Guido CERRI ${ }^{1}$

Correspondência para:

Cibele Figueira Carvalho

Travessa Leon Berry, 122 - Cep: 01402-030

-SãoPaulo-SP cibelefcarvalho@terra.com.br

Recebido para publicação: 17/12/2005 Aprovado para publicação: 22/11/2007

\title{
Ultra-sonografia dúplex-Doppler na avaliação morfológica e hemodinâmica das artérias aorta e mesentérica cranial em cães
}

1 - Faculdade de Medicina da Universidade de São Paulo, São Paulo - SP

2 - Faculdade de Medicina Veterinária da Universidade de São Paulo, São Paulo - SP

Resumo

O conhecimento dos sinais normais ao ultra-som Doppler de cada vaso sanguíneo é fundamental na sua identificação. Existem poucos artigos na literatura veterinária que tratam do aspecto normal dos vasos abdominais nos cães. Com o objetivo de verificar a viabilidade técnica da aplicação do US-Doppler na avaliação da aorta abdominal e da artéria mesentérica cranial; vinte cães normais foram submetidos a este exame sem sedação. Foi descrito e avaliado o padrão de mapeamento dúplex-Doppler colorido destes vasos e os resultados comparados com aqueles descritos em literatura. Embora encontradas algumas limitações técnicas, verificamos que o US-Doppler pode ser aplicado com sucesso como meio não invasivo para a detecção de alterações no fluxo sanguíneo mesentérico em cães sem a necessidade de submete-los a contenção química.

\section{Introdução}

O ultra-som Doppler (US-Doppler) é um método relativamente novo dentro da ultra-sonografia veterinária em pequenos animais. A ultra-sonografia com dúplexDoppler colorido fornece informações anatômicas e dinâmicas em tempo real. Durante os exames podem ser determinadas a presença e a direção do fluxo sanguíneo de um vaso e suas características hemodinâmicas.

A ultra-sonografia modo-B é ideal para detectar alteração na topografia e arquitetura dos vasos (por exemplo, no caso de fístulas ou "shunts"), para medir o diâmetro dos mesmos, para mensurar a espessura e regularidade das paredes vasculares, ou ainda identificar a presença de estruturas anormais perivasculares ou intraluminais (por exemplo, trombos ou tumores). No entanto, sabe-se que um trombo recente pode ser anecogênico como o próprio sangue. ${ }^{1}$ A pulsação das artérias também pode ser observada, mas deve-se considerar que a pulsação de uma artéria pode influenciar uma veia adjacente, dependendo de seu calibre e topografia. ${ }^{1,2}$ Os vasos sanguíneos abdominais têm uma estrutura tubular de paredes bem definidas em plano longitudinal. As paredes são paralelas hiperecogênicas e têm uma aparência linear fina. Os vasos quando não submetidos à compressão aparecem em plano transversal com aspecto oval ou circular. O lume vascular é anecogênico devido a ausência de eco no seu interior. Quando o fluxo sanguíneo é lento e o diâmetro de um vaso é grande o suficiente, podem-se ver os ecos que se movimentam e correspondem ao fluxo sanguíneo. ${ }^{1,2,3,4}$

O conhecimento dos sinais normais ao Ultra-som Doppler de cada vaso sanguíneo é importante na sua identificação, pois o sinal Doppler é específico para cada tipo de vaso bem como varia com sua topografia. Reconhecer as alterações no espectro Doppler somente é possível se o ultra-sonografista conhecer também as variações da normalidade.

A melhor imagem ultra-sonográfica da aorta abdominal em sua porção caudal é 
obtida com o animal em decúbito lateral direito. O transdutor é colocado na região caudodorsal do abdome, onde a pulsação da aorta pode ser observada longitudinalmente em posição ventral às vértebras lombares, à esquerda e paralela ao trajeto da veia cava caudal. A parte cranial da aorta abdominal (cranial à artéria frenicoabdominal esquerda) não é de fácil visibilização, especialmente em cães com tórax profundo, devido aos artefatos ocasionados pelo gás no trato gastrintestinal e pelas costelas. Às vezes, quando há muito gás pode ser mais fácil obter uma imagem sagital melhor da região cranial da aorta em decúbito dorsal, medialmente ao rim direito. Ao mapeamento Doppler, a aorta possui um perfil típico de velocidade de fluxo laminar do tipo achatado e a morfologia da onda é típica de um fluxo de padrão de alta impedância ou resistência. Possui pico sistólico afilado com uma janela espectral larga e bem definida. Isto ocorre porque a distribuição de velocidade é estreita através do lume vascular, ou seja, a grande maioria das células sanguíneas move-se a uma velocidade uniforme. O pico sistólico é seguido por uma onda de fluxo retrógrado e corresponde ao efeito de desaceleração momentânea devido a resistência vascular periférica e a elasticidade destes vasos. Depois pode ser visto uma onda de fluxo anterógrada que representa o momento protodiastólico. $\mathrm{O}$ momento final da diástole é o período ideal para se avaliar a resistência vascular. Se houver uma pausa maior entre duas contrações ventriculares, podemos ainda observar ondas adicionais com velocidades mais baixas. $^{5}$

A artéria mesentérica cranial é o segundo ramo ímpar da aorta abdominal. ${ }^{1,6}$ Este vaso supre sangue para o duodeno, todo o intestino delgado e lado direito do cólon. ${ }^{7}$ Situa-se ligeiramente cranial a origem da artéria renal direita e caudal a origem do tronco celíaco. Com o animal em decúbito dorsal, o tronco celíaco e a artéria mesentérica cranial apresentam ao exame ultra-sonográfico uma imagem em forma de "V" no plano de varredura sagital.
Quando o cão é colocado em decúbito lateral direito, o tronco celíaco e a artéria mesentérica cranial seguem em trajeto paralelo até que se aproximam neste ponto. Pode-se observar ao mapeamento Doppler espectral um padrão de fluxo de resistência intermediária. O pico sistólico é mais amplo com uma janela espectral pequena. Após o pico sistólico, a velocidade diminui bruscamente, o fluxo torna-se mais rápido, depois fica mais lento novamente. O sangue tem fluxo de alta resistência durante o jejum, e baixa resistência no período pós-prandial. ${ }^{5}$ Isto é explicado devido ao aumento do fluxo sanguíneo no estômago, duodeno e pâncreas no período pós prandial.

O exame ultra-sonográfico com Doppler colorido e o de amplitude é utilizado com freqüência em medicina humana com a finalidade de avaliar vasos mesentéricos para determinar as características hemodinâmicas dos mesmos em período de jejum e no pós prandial. Além destas investigações em pacientes normais, o US Doppler tem sido utilizado para detectar e quantificar as alterações hemodinâmicas no fluxo sanguíneo mesentérico em casos de doenças do sistema digestório como a hipertensão portal, a doença de Crohn e a isquemia mesentérica. ${ }^{8}$ Nestes casos é possível verificar a viabilidade de segmentos de alça intestinal isquêmicos e determinar a extensão do segmento inviável que deverá ser retirado cirurgicamente. ${ }^{7} \mathrm{O}$ papel do dúplex-Doppler na avaliação da artéria mesentérica cranial não está bem definido até o momento. Estes resultados vagos devem-se em parte à dificuldade de visibilizar adequadamente esses vasos. A superposição de alças intestinais e a obesidade dos pacientes humanos são fatores primários limitantes ao acesso dos feixes sonoros, sendo que sob excelentes condições somente as porções dos troncos principais são demonstradas. ${ }^{7} \mathrm{Na}$ veterinária somandose a estes fatores podemos considerar ainda a dificuldade de contenção dos animais, tornando mais difícil o exame de rotina.

Existem poucos artigos na literatura veterinária que tratam do aspecto normal dos 
vasos abdominais nos cães. ${ }^{1,2,5}$ Encontramos relatos que descrevem resultados dos exames de avaliação com Ultra-som Doppler renal ${ }^{9}$, hepático ${ }^{10,11,12,13}$, prostático $^{14}$, das artérias fetais e maternas ${ }^{15}$, assim como o aspecto da veia porta ${ }^{11}$, hepática ${ }^{9}$ em cães saudáveis ou com várias doenças. As afecções gastrointestinais são freqüentes nos cães. Embora a ultra-sonografia seja um meio de diagnóstico de rotina nos animais com doenças gastrointestinais, é escassa a literatura internacional no que se refere a avaliação hemodinâmica dos vasos mesentéricos e abdominais que o ultra-som Doppler pode fornecer nestes casos. Entretanto é possível encontrar trabalhos com a descrição de padrões hemodinâmicos e a interferência do jejum ${ }^{8}$ e da composição dos alimentos ${ }^{6}$ no processo de digestão e o aspecto hemodinâmicos dos vasos esplâncnicos em animais com enteropatias crônicas ${ }^{16}$ e sensibilidade alimentar ${ }^{17}$, assim como a influência de processos que levam a anemia no cão ${ }^{18,19}$.

Este trabalho teve como objetivo descrever a técnica e verificar a viabilidade da aplicação do US-Doppler na avaliação das artérias aorta e mesentérica cranial em cães normais não sedados, contribuindo assim, para estabelecer parâmetros de normalidade e eventuais variações da mesma.

\section{Material e Método}

Procedeu-se um estudo prospectivo no período de junho a dezembro de 2004. Foram realizados exames de ultra-som Doppler em cães adultos de idades variadas, ambos os sexos, com ou sem raça definida que atendessem aos critérios de inclusão estabelecidos no estudo. Parte dos exames foi realizado no Setor de Ultra-sonografia do Hospital Veterinário da Faculdade de Medicina Veterinária e Zootecnia da Universidade de São Paulo e outra parte no Setor de Ultra-sonografia do Centro de Especialidades PROVET - Moema. Consideraram-se como critérios de inclusão animais provindos da rotina hospitalar do serviço de ultra-sonografia do HOVET da
Faculdade de Medicina Veterinária e Zootecnia da Universidade de São Paulo ou do setor de ultra-sonografia da rotina do PROVET selecionados como sadios por meio de exame clínico, perfil laboratorial hepático e exame ultra-sonográfico da cavidade abdominal em modo-B sem alterações.

\section{Equipamento}

Foi utilizado um equipamento modoB da marca ATL $\AA$, modelo HDI $5000 \mathrm{com}$ transdutores multifrequenciais convexos de banda variável de 2,0 a 5,0 MHz, e 4,0 a 7,0 $\mathrm{MHz}$ e transdutor linear de $5 \mathrm{a} 12 \mathrm{MHz}$. As imagens foram registradas em termo impressora modelo UP-890MD da marca SONY ${ }^{\circledR}$ no Hospital Veterinário da Faculdade de Medicina Veterinária e Zootecnia da Universidade de São Paulo.

Foi utilizado também um equipamento modo-B da marca GE ${ }^{\circledR}$, modelo LOGIC3 com transdutores multifrequenciais convexos de banda variável de 4 a $6 \mathrm{MHz}$ e transdutor linear de 7 a 10 $\mathrm{MHz}$. As imagens foram registradas em termo impressora modelo UP-D895MD da marca SONY® no PROVET.

\section{Método}

Em uma primeira etapa estes animais foram submetidos ao preparo prévio intestinal 24 a 48 horas antes do exame com antifiséticos (Luftal ${ }^{\circledR}$ : dimeticona) e submetidos a jejum de sólidos no mínimo de 8 horas para a realização dos exames ultra-sonográficos.

Em todos os animais foi realizada ampla tricotomia abdominal, incluindo os músculos epaxiais dorsalmente, a pelve caudalmente e o processo xifóide cranialmente.

Para a realização da avaliação ultrasonográfica foi necessário a utilização de gel para contato em toda a região a ser examinada. Todos os exames foram realizados pelo mesmo operador.

Após o exame ultra-sonográfico do abdome total e uma vez descartados quaisquer alterações, foram realizadas as 
medidas e avaliação Doppler dos vasos em questão, ou seja, da artéria aorta próximo a artéria renal e da artéria mesentérica cranial próximo a sua origem. Foi estabelecido o limite máximo de 20 minutos para a realização do exame ultra-sonográfico completo.

Todos os dados foram protocolados e dispostos em quadros para análise estatística. Com os dados obtidos efetuamos correlações utilizando o coeficiente de Pearson (r) com índice de significância (p) maior ou igual a 0,05 . Foram considerados normais os valores comparados com os obtidos em literatura. ${ }^{5,8}$

\section{Técnica de Exame}

O animal foi posicionado em decúbito lateral direito com o auxílio do proprietário e foi contido mecanicamente na mesa de exame. Com o transdutor na parede lateral esquerda do abdome, próximo à margem caudal das costelas localizou-se o rim esquerdo e posicionando o transdutor medial e cranialmente identificou-se a artéria aorta abdominal. Acompanhando-a em plano longitudinal em direção cranial pode ser identificada a origem da artéria mesentérica cranial. Mensuramos o diâmetro de ambos os vasos. Para conseguir uma perfeita avaliação dos parâmetros hemodinâmicos do vaso foi realizada uma inspeção sistemática do mesmo até se conseguir o paralelismo do vaso em relação ao transdutor associado a um ângulo de insonação menor que $60^{\circ}$. O PRF e o filtro foram ajustados até a melhor caracterização de amplitude do sinal sem que houvesse artefato de ambigüidade. Usou-se o modo colorido para auxiliar na identificação dos vasos e determinar a presença ou ausência de fluxo sanguíneo nos mesmos. Os parâmetros coloridos foram ajustados para que o lume do vaso estivesse preenchido somente com uma cor (isto é sem ambigüidade de sinal detectado) e a informação colorida não ultrapassasse o lume vascular, mas que o mesmo estivesse preenchido com cor. $\mathrm{O}$ volume de amostra foi fixado em $2 \mathrm{~mm}$, colocado na porção central do vaso e acionado o Doppler pulsado. Desta forma foi obtido um traçado nesta região. Quando o traçado apresentouse livre de artefatos, corrigiu-se o ângulo de insonação e congelou-se a imagem para a análise da morfologia das ondas. Sinteticamente, podemos dizer que para a avaliação morfológica e hemodinâmica dos vasos verificamos:

- parâmetros morfométricos: diâmetro dos vasos (d) pré-prandial

- parâmetros velocimétricos do fluxo: velocidade de pico sistólico (VPS) préprandial e velocidade diastólica final (VDF) pré-prandial

- índices hemodinâmicos de impedância: índice de resistividade (RI)

\section{Resultados}

Foram selecionados 20 cães sadios de raça, sexo, idade e pesos variados conforme descrito no quadro 1 . O peso dos animais variou de 2,00 a 25,00 kg, com média de $10,75 \pm 7,50 \mathrm{~kg}$.

A avaliação da morfologia das ondas obtidas com o mapeamento dúplex Doppler colorido pode ser realizada nos animais de número $01,02,03,04,08,15$, $16,17,18,19$ e $20 . \mathrm{Na}$ aorta observamos um perfil de fluxo laminar do tipo achatado e morfologia da onda de alta resistência. Verificamos a presença de picos sistólicos afilados com janela espectral larga e bem definida e distribuição de velocidade estreita. O pico sistólico apresentou-se seguido por uma onda de fluxo retrógrado, e em seguida uma onda de fluxo anterógrada (Figura 1). $\mathrm{Na}$ artéria mesentérica cranial (Figura 2) pode-se observar padrão de fluxo de resistência intermediária. O pico sistólico apresentou-se pouco mais amplo com uma janela espectral pequena. Após o pico sistólico, a velocidade diminuiu bruscamente, o fluxo tornou-se mais rápido e depois mais lento novamente (Figura 3).

Os resultados das mensurações dos diâmetros (em centímetros) e das velocidades 
Quadro 1 - Relação dos animais segundo a raça, sexo, idade e peso. São Paulo - 2005

\begin{tabular}{|c|c|c|c|c|}
\hline Animal & raça & sexo & idade & Peso (kg) \\
\hline 01 & SRD & macho & 5 anos & 15 \\
\hline 02 & Whippet & macho & 10 anos & 16 \\
\hline 03 & Teckel & fêmea & 6 anos & 6 \\
\hline 04 & Teckel & fêmea & 1 ano & 7 \\
\hline 05 & Boxer & macho & 6 anos & 17 \\
\hline 06 & Rottweiler & macho & 5 anos & 25 \\
\hline 07 & Poodle & fêmea & 7 anos & 6 \\
\hline 08 & Schnauzer & fêmea & 1 ano & 7 \\
\hline 09 & Pitbull & fêmea & 2 anos & 25 \\
\hline 10 & Maltes & fêmea & 4 anos & 3 \\
\hline 11 & Cocker & macho & 4 anos & 12 \\
\hline 12 & Spitz Alemão & fêmea & 1 ano & 2 \\
\hline 13 & SRD & macho & 2 anos & 10 \\
\hline 14 & SRD & macho & 2 anos & 22 \\
\hline 15 & Spitz Alemão & fêmea & 1 ano & 2 \\
\hline 16 & Poodle & fêmea & 2 anos & 3 \\
\hline 17 & Poodle & macho & 1 ano & 4 \\
\hline 18 & Poodle & macho & 2 anos & 5 \\
\hline 19 & Cocker & fêmea & 3 anos & 13 \\
\hline 20 & SRD & macho & 3 anos & 15 \\
\hline
\end{tabular}

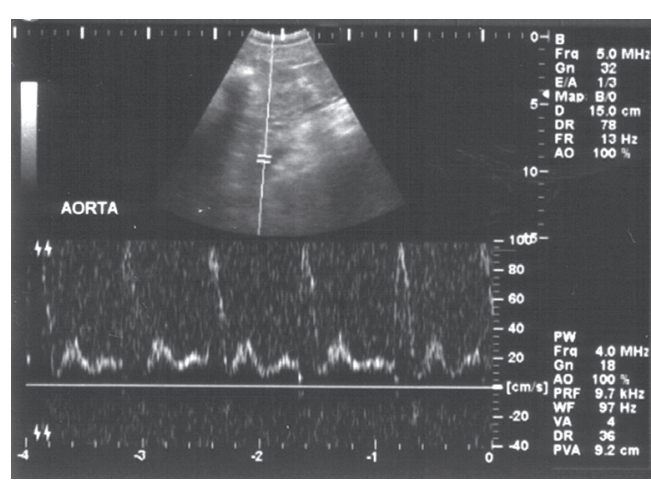

Figura 1 - Imagem dúplex-Doppler da artéria aorta abdominal e respectivo traçado espectral onde notamos fluxo sanguíneo laminar com morfologia característica e de padrão trifásico

obtidas ao exame de ultra-som dúplexDoppler colorido das artérias aorta abdominal e mesentérica cranial encontramse descritos no quadro 2. Com os dados obtidos efetuamos correlações utilizando o coeficiente de Pearson (r) com índice de significância (p) maior ou igual a 0,05.

Considerando-se os cães examinados, independente do porte do animal a amostra geral indicou que o diâmetro da aorta variou entre $0,43 \mathrm{~cm}$ e $0,92 \mathrm{~cm}$. A média do diâmetro encontrado foi $0,65 \pm 0,17 \mathrm{~cm}$. A velocidade de pico sistólico (VPS) da aorta variou entre $44,00 \mathrm{~cm} / \mathrm{s}$ e $135,18 \mathrm{~cm} / \mathrm{s}$. A média de velocidade foi $95,49 \pm 35,43 \mathrm{~cm} /$

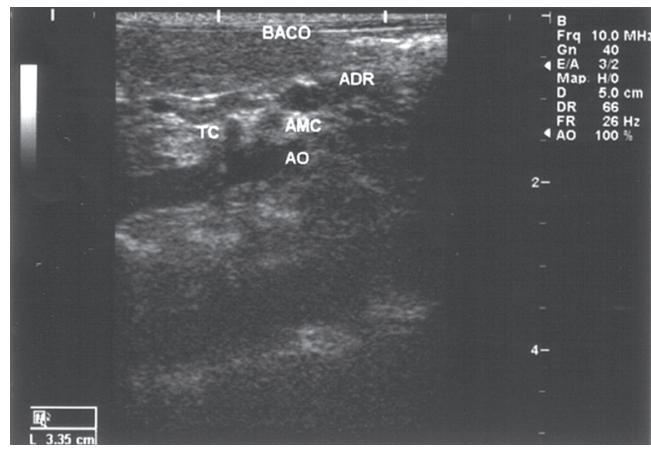

Figura 2 - Imagem em modo-B em plano longitudinal da aorta abdominal (AO) e seus ramos: artéria mesentérica cranial (AMC) e tronco celíaco (TC). Observa-se ainda a parte da imagem do baço e a adrenal esquerda (ADR) sem evidências sonográficas de alterações

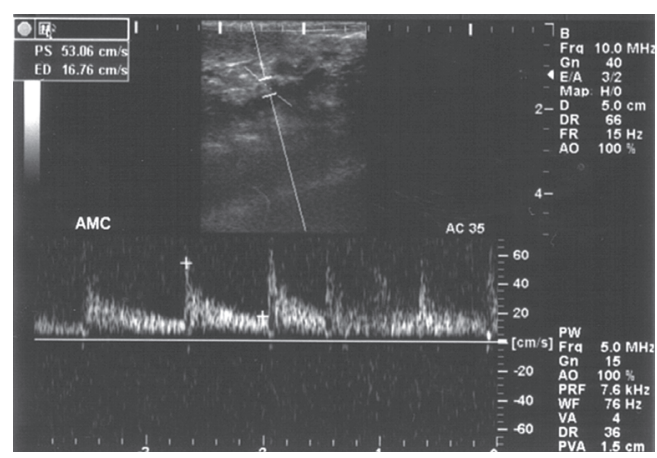

Figura 3 - Imagem duplex-Doppler da artéria mesentérica cranial (AMC) mostrando o vaso em modo-B e o traçado com padrão de fluxo de resistência intermediária. 
Quadro 2 - Resultados das mensurações em cm e de velocidades obtidas ao exame de ultra-som dúplex-Doppler das artérias aorta abdominal e mesentérica cranial dos cães avaliados. São Paulo - 2005

\begin{tabular}{|c|c|c|c|c|c|c|c|c|c|c|c|c|c|c|c|c|c|c|c|c|}
\hline Animais & 01 & 02 & 03 & 04 & 05 & 06 & 07 & 08 & 09 & 10 & 11 & 12 & 13 & 14 & 15 & 16 & 17 & 18 & 19 & 20 \\
\hline $\begin{array}{c}\text { Diâmetro } \\
\mathrm{d} \text { aorta } \\
(\mathrm{cm})\end{array}$ & 0,85 & 0,92 & 0,55 & 0,60 & 0,86 & 0,90 & 0,80 & 0,60 & 0,80 & 0,43 & 0,56 & 0,45 & 0,60 & 0,66 & 0,43 & 0,45 & 0,48 & 0,80 & 0,56 & 0,65 \\
\hline $\begin{array}{l}\text { VPS- } \\
\text { Aorta } \\
(\mathrm{cm} / \mathrm{s})\end{array}$ & 104,2 & 101,3 & 123,1 & 135,2 & 102,1 & 126,3 & 95,5 & 128,3 & 118,4 & 68,4 & 53,0 & 46,5 & 132,6 & 135,8 & 45,3 & 44,0 & 48,8 & 120,0 & 52,7 & 128,4 \\
\hline $\begin{array}{l}\text { VDF- } \\
\text { Aorta } \\
(\mathrm{cm} / \mathrm{s})\end{array}$ & 77,2 & 76,2 & 83,2 & 84,4 & -- & - & -- & 83,1 & - & -- & -- & - & - & -- & -- & -- & - & - & -- & -- \\
\hline $\begin{array}{c}\text { Diàmetro } \\
\text { da artéria } \\
\text { meserténic } \\
\text { a cranial } \\
(\mathrm{cm})\end{array}$ & 0,35 & 0,40 & 0,21 & 0,28 & 0,40 & 0,38 & 0,32 & 0,27 & 0,34 & 0,30 & 0,31 & 0,28 & 0,36 & 0,34 & 0,28 & 0,25 & 0,28 & 0,37 & 0,30 & 0,35 \\
\hline $\begin{array}{l}\text { VPS- } \\
\text { AMC } \\
(\mathrm{cm} / \mathrm{s})\end{array}$ & 58,1 & 117,3 & 102,1 & 102,3 & -- & - & -- & 58,41 & - & 46,8 & -- & - & - & -- & 48,7 & 44,9 & 46,9 & 64,78 & 49,8 & 54,1 \\
\hline $\begin{array}{l}\text { VDF- } \\
\text { AMC } \\
\left(\mathrm{cm}^{\prime} \mathrm{s}\right)\end{array}$ & 16,0 & 36,1 & 30,2 & 45,8 & -- & -- & -- & 16,0 & -- & 15,82 & -- & -- & -- & -- & 12,2 & 15,6 & 16,7 & 21,59 & 13,42 & 18,90 \\
\hline
\end{tabular}

s. A velocidade diastólica final (VDF) variou entre $76,20 \mathrm{~cm} / \mathrm{s}$ e $84,40 \mathrm{~cm} / \mathrm{s}$. A média calculada foi $80,82 \pm 3,81 \mathrm{~cm} / \mathrm{s}$. O diâmetro da artéria mesentérica cranial variou entre $0,21 \mathrm{~cm}$ e $0,40 \mathrm{~cm}$. A média do diâmetro foi $0,32 \pm 0,05 \mathrm{~cm}$. A VPS variou entre 44,90 $\mathrm{cm} / \mathrm{s}$ e $117,30 \mathrm{~cm} / \mathrm{s}$, sendo a média calculada em 66,19 $\pm 25,69 \mathrm{~cm} / \mathrm{s}$. A VDF variou entre $12,20 \mathrm{~cm} / \mathrm{s}$ e $45,80 \mathrm{~cm} / \mathrm{s}$. A média encontrada foi $21,53 \pm 10,39 \mathrm{~cm} / \mathrm{s}$.

\section{Discussão e Conclusões}

Em todos os animais examinados foi possível identificar os vasos e mensurar o diâmetro dos mesmos utilizando imagem em modo-B com o auxílio do Doppler colorido. Este fato sugere que o mapeamento colorido auxilia na identificação dos vasos, e que mesmo sem a utilização de contenção química nos animais, a técnica de varredura descrita é reproduzível e capaz de fornecer imagens adequadas para esta finalidade. Observou-se que os diâmetros da aorta e da artéria mesentérica cranial aumentam à medida que aumenta $\mathrm{O}$ peso do animal. Ao aplicar testes estatísticos notamos que existe uma correlação positiva entre o peso e o diâmetro destes vasos, conforme já foi descrito em literatura. ${ }^{6}$ É importante ressaltar que neste estudo não havia dentre os animais da amostra cães obesos ou caquéticos. Os resultados obtidos foram semelhantes aos encontrados na literatura veterinária que descrevem o diâmetro da aorta nesta porção variando em torno de $0,60 \mathrm{~cm}$ a $1,10 \mathrm{~cm}$ com média de
$0,80 \mathrm{~cm}^{6}$ ou ainda $0,990,14 \mathrm{~cm}^{8}$. Os resultados obtidos quanto ao diâmetro da artéria mesentérica cranial também estão em acordo com a literatura que cita uma variação entre 0,20 e $0,60 \mathrm{~cm}$ com média de $0,32 \mathrm{~cm}^{6}$ ou $0,48 \mathrm{~cm} \pm 0,06 \mathrm{~cm}^{8}$. Há trabalhos que afirmam que a acurácia das medidas dos diâmetros dos vasos abdominais de menor calibre é questionável, pois é considerado uma potencial fonte de erros na ultra-sonografia modo-B ${ }^{8}$. No entanto estudos no homem indicam que não há ou há mínimas alterações no diâmetro da artéria mesentérica superior no período pré e pós-prandial. ${ }^{5,7} \mathrm{Em}$ oito cães $(8 / 20$ ou $40 \%$ examinados não foi possível a obtenção de imagens adequadas ao mapeamento dúplex-Doppler da artéria mesentérica dentro do tempo de exame estipulado (20 minutos como tempo máximo). Estudos em cães mostram que o estresse da contenção e outros fatores ambientais poderiam ocasionar liberação de vasopressina e promover uma vasoconstrição no leito vascular esplâncnico. ${ }^{8,16}$ É possível que estas alterações possam ter ocorrido nesses animais que se apresentaram mais estressados, com alteração de padrão respiratório e outras manifestações comportamentais de estresse. Estipular um tempo máximo de duração do exame auxilia na determinação do índice de falha técnica do mesmo. As principais causas da falha técnica ou não obtenção de traçado espectral adequado neste estudo foram: temperamento do animal dificultando a contenção mecânica do mesmo, taquipnéia 
e presença de gases em trato gastrintestinal. Outros fatores técnicos que devem ser considerados na avaliação hemodinâmica com US-Doppler são o tamanho do vaso e a posição do transdutor. Em animais de porte muito pequeno fica mais difícil posicionar e manter a janela adequada para obter o traçado da onda. Quando o transdutor não fica posicionado adequadamente, não obtemos o ângulo de insonação ideal, impossibilitando a avaliação hemodinâmica correta do vaso em questão. Embora todos os exames tenham sido feitos por um único operador, notamos que ao início do projeto havia uma maior dificuldade para se obter o plano de imagem e a angulação ideal para o mapeamento Doppler espectral da artéria mesentérica cranial, com isto podemos inferir ainda que a habilidade e a experiência do ultrasonografista podem influenciar na avaliação desses vasos abdominais de menor calibre.

$\mathrm{Na}$ aorta abdominal observou-se fluxo sanguíneo laminar com morfologia da onda de padrão trifásico e na artéria mesentérica cranial observamos fluxo de resistência intermediária conforme citado em literatura. ${ }^{5} \mathrm{Na}$ literatura veterinária não encontramos dados sobre a velocidade média deste vaso.

Avaliando-se os resultados estatísticos, notou-se uma correlação positiva entre o peso do animal e a VPS da aorta. Notamos também uma correlação negativa entre o peso dos animais da amostra e a velocidade diastólica final da aorta, ou seja, podemos inferir que a medida que aumenta o diâmetro deste vaso, conforme o porte do animal, diminui a velocidade diastólica final do fluxo sanguíneo no mesmo. Isto não acontece com a artéria mesentérica cranial. Talvez pela pouca variação do diâmetro da mesma independente do porte do animal examinado, a velocidade do sangue neste vaso independe do diâmetro do mesmo e ou do porte do animal. Ao calcularmos ainda a correlação entre as velocidades de pico sistólico da aorta e da artéria mesentérica cranial observamos que quanto maior a VPS da aorta, tanto maior a VPS da artéria mesentérica cranial, mostrando uma relação significante entre os valores hemodinâmicos destes vasos. Uma vez que este trabalho demonstra resultados de um estudo inédito, podemos dizer que ainda há que se pesquisar mais nesta área. Acreditamos que isto seja especialmente útil na avaliação dos shunts portossistêmicos, nos casos de hipertensão portal não cirrótica, em animais com alterações isquêmicas devido a presença de possíveis trombos ou ainda em casos de animais com formações neoplásicas abdominais. Porém mediante os resultados podemos concluir que o ultra-som Doppler pode ser aplicado com sucesso como um meio não invasivo para detecção de alterações no fluxo mesentérico em cães, embora haja limitações técnicas como este trabalho demonstrou.

\section{Duplex-Doppler ultrasonography for morfologycal and hemodynamic evaluation of aorta and cranial mesenteric arteries in dogs}

\begin{abstract}
The knowledge of the normal Doppler signs of each blood vessel is important in their identification. There are few reports in veterinary literature that describe the normal aspect of abdominal blood vessels in dogs. The aim of this paper was verify technical viability of US Doppler use to assessment of the abdominal aorta and cranial mesenteric arteries; twenty clinically normal unsedated dogs were examinated. It was described and assessed characteristic duplex color Doppler spectrum of each blood vessel and the results were compared with that found in literature. Although some technical limitations
\end{abstract}

Key words: Ultrasonography. Doppler. Dogs. 
were found, US Doppler could be successfully used as a noninvasive method to detect mesenteric blood flow changes in dogs without chemical contention.

\section{Referências}

1 SPAULDING, K. A. A review of sonographic identification of abdominal blood vessels and juxtavascular organs. Veterinary Radiology and Ultrasound, v. 38, n. 1, p. 4-23, 1997.

2 FINN-BODNER, S. T.; HUDSON, J. A. Abdominal vascular sonography. Veterinary Clinics of North America Small Animal Practice, v. 28, n. 4, p. 887942, 1998.

3 KIRCHER, P. et al. Influence of food composition in unsedated normal dogs: a Doppler study. Veterinary Journal, v. 166, n. 3, p. 265-272, 2003.

4 NYLAND, T. G.; MATTON, J. S. Small animal diagnostic ultrasound. $2^{\text {nd }}$ ed. Philadelphia: W.B. Saunders, 2002. $461 \mathrm{p}$

5 SZATMÁRI, V.; SÓTONYI, P.;VÖROS, K. Normal duplex Doppler waveforms of major abdominal blood vessels in dogs: a review. Veterinary Radiology and Ultrasound, v. 42, n. 2, p. 93-107, 2001.

6 KAMIKAWA, L. Avaliação ultra-sonográfica da aorta abdominal e seus ramos em cães. 2003. 87 f. Dissertação (Mestrado) - Faculdade de Medicina Veterinária e Zootecnia, Universidade de São Paulo, São Paulo, 2003.

7 CERRI, G. G.; MÓLNAR, L. J.; VEZOZZO, D. C. P. Doppler. São Paulo: Sarvier, 1998. cap. 6, p. 120-121.

8 RIESEN, S. et al. Doppler measurement of splanchnic blood flow during digestion in unsedated normal dogs. Veterinary Radiology and Ultrasound, v. 43, n. 6, p. 554-560, 2002.

9 MORROW, K. L. et al. Comparison of the resistive index to clinical parameters in dogs with renal disease. Veterinary Radiology and Ultrasound, v. 37, n. 3, p. 193-199, 1996.

10 KANTROWITZ, B. M.; NYLAND, T. G.; FISHER, $P$. Estimation of portal blood flow using duplex realtime and pulsed Doppler ultrasound imaging in the dog. Veterinary Radiology and Ultrasound, v. 30, n. 5, p. 222-226, 1989.

11 LAMB, C. R.; MAHONEY, P. N. Comparison of three methods for calculating portal blood flow velocity in dogs using duplex-Doppler ultrasonography. Veterinary Radiology and Ultrasound, v. 35, n. 3, p. 190-194, 1994.

12 LAMB, C. R.; BURTON, C. A.; CARLISLE, C. H. Doppler measurement of hepatic arterial flow in dogs: technique and preliminary findings. Veterinary Radiology and Ultrasound, v. 40, n. 1, p. 77-81, 1999. $13 \mathrm{WU}$, J. X.; CARLISLE, C. H. Ultrasonographic examination of the canine liver based on recognition of hepatic and portal veins. Veterinary Radiology and Ultrasound, v. 36, n. 3, p. 234-239, 1995.

14 NEWLL, S. M. et al Doppler ultrasound of the prostate in normal dogs and in dogs with cronic lymphocytic-lymphoplasmocytic prostitis. Veterinary Radiology and Ultrasound, v. 39, n. 4, p. 332-336, 1998.

15 NAUTRUP, C. P. Doppler ultrasonography of canine maternal and fetal arteries during normal gestation. Journal of Reproduction and Fertility, v. 112, n. 2, p. 301-314, 1998.

16 GASCHEN, L. et al. Pattern recognition and feature extraction of canine celiac and cranial mesenteric arterial waveforms: normal versus chronic enteropathy - a pilot study. Veterinary Journal, v. 169 , n. 2, p. 242-250, 2005.

17 KIRCHER, P. et al. Doppler ultrasonographic evaluation of gastrointestinal hemodynamics in food hypersensivities: a canine model. Journal of Veterinary Internal Medicine, v. 18, n. 5, p. 605-611, 2004.

18 KOMA, L. M. et al. Comparison of effects of uncomplicated canine babesiosis and canine normovolemic anaemia on abdominal splanchnic Doppler characteristics - a preliminary investigation. Journal of South Africa Veterinary Association, v. 76, n. 3, p. 138-145, 2005

19 KOMA, L. M. et al. Influence of normovolemic anemia on Doppler characteristics of the abdominal aorta and splanchnic vessels in Beagles. American Journal of Veterinary Research, v. 66, n. 2, p. 187-195, 2005.

20 AN, Y. J. et al. Application of pulsed Doppler ultrasound for the evaluation of small intestinal motility in dogs. Journal of Veterinary Science, v. 2, n. 1, p. 7174, 2001.

21 CLAUDICE-ENGLE, T. et al. Power Doppler imaging of focal lesions of the gastrointestinal tract: comparison with conventional color Doppler imaging. Journal of Ultrasound Medicine, v. 15, n. 1, p. 63-66, 1996.

22 KAWAKAMA, J.; KODAIRA, S.; CERRI, G. G. Física. In: CERRI, G. G.; ROCHA, D. C. Ultra-sonografia abdominal. São Paulo: Sarvier, 1993. cap. 1, p. 1-14.

23 NYLAND, T. G. et al. Diagnosis of urinary tract obstruction in dogs using duplex Doppler ultrasonography. Veterinary Radiology and Ultrasound, v. 34, n. 5 , p. 384-352, 1993.

24 YANIK, L. The basics of Doppler ultrasonography. Veterinary Medicine, v. 97, n. 5, p. 388-400, 2002. 